\title{
Temperature Compensation Fiber Bragg Grating Pressure Sensor Based on Plane Diaphragm
}

\author{
Minfu LIANG, Xinqiu FANG ${ }^{*}$, and Yaosheng NING \\ Key Laboratory of Deep Coal Resource Mining, Ministry of Education of China, School of Mines, China University of \\ Mining and Technology, Xuzhou, 221116, China \\ *Corresponding author: Xinqiu FANGＥ-mail address: fangxinqiu@cumt.edu.cn
}

\begin{abstract}
Pressure sensors are the essential equipments in the field of pressure measurement. In this work, we propose a temperature compensation fiber Bragg grating (FBG) pressure sensor based on the plane diaphragm. The plane diaphragm and pressure sensitivity FBG (PS FBG) are used as the pressure sensitive components, and the temperature compensation FBG (TC FBG) is used to improve the temperature cross-sensitivity. Mechanical deformation model and deformation characteristics simulation analysis of the diaphragm are presented. The measurement principle and theoretical analysis of the mathematical relationship between the FBG central wavelength shift and pressure of the sensor are introduced. The sensitivity and measure range can be adjusted by utilizing the different materials and sizes of the diaphragm to accommodate different measure environments. The performance experiments are carried out, and the results indicate that the pressure sensitivity of the sensor is $35.7 \mathrm{pm} / \mathrm{MPa}$ in a range from $0 \mathrm{MPa}$ to $50 \mathrm{MPa}$ and has good linearity with a linear fitting correlation coefficient of $99.95 \%$. In addition, the sensor has the advantages of low frequency chirp and high stability, which can be used to measure pressure in mining engineering, civil engineering, or other complex environment.
\end{abstract}

Keywords: Fiber Bragg grating; plane diaphragm; temperature compensation; pressure sensor; performance experiment; low frequency chirp

Citation: Minfu LIANG, Xinqiu FANG, and Yaosheng NING, "Temperature Compensation Fiber Bragg Grating Pressure Sensor Based on Plane Diaphragm," Photonic Sensors, 2018, 8(2): 157-167.

\section{Introduction}

Pressure sensors are the essential equipments in the field of pressure measurement. But for electrical pressure sensors have a lot of technological limitations which restrict their application in harsh environments with electromagnetic interference, dangerous explosion matters, or extreme temperatures and pressures [1]. While the fiber Bragg grating (FBG) sensing technology being as a new measurement technology [2], which is mainly used as a sensitive component for the manufacture of optical fiber sensors and has the virtues of intrinsic safety, small size, light weight, anti-causticity, anti-electromagnetic, high sensitivity, strong reliability, and ease to form a quasi-distributed sensing system [3]. With these merits and different packaging structures, many physical quantities for measurement of the temperature, strain, pressure, torque, and others can

Received: 29 March 2017 / Revised: 17 February 2018

(C) The Author(s) 2018. This article is published with open access at Springerlink.com

DOI: $10.1007 / \mathrm{s} 13320-018-0417-9$

Article type: Regular 
be achieved [4].

However, the bare FBG is thin and brittle, and its usual pressure sensitivity is only $0.003 \mathrm{~nm} / \mathrm{MPa}$ [5], which cannot meet the requirements of engineering application and must adopt the appropriate packaging technology and methods in the actual measurement. Peng et al. [6] proposed an FBG pressure sensor based on the free elastic cylinder and obtained the pressure sensitivity of $27 \mathrm{pm} / \mathrm{MPa}$. Zhang et al. [7] reported a pressure sensor by using the polymer coated FBG in the thick-wall metal elastic cylinder. Their results showed the pressure sensitivity of this sensor is 1722 times higher than that of the bare grating. Some other groups used methods contain bonding FBGs to an isosceles triangle cantilever or a diaphragm structure [8-10] or embedding FBG in polymer [11, 12]. However, some methods have complex structures which are hard to be fabricated and multiplexed in one single optic fiber.

In order to improve the strain-temperature cross-sensitivity of the FBG, Pan et al. [13] described a distinguishing range FBG pressure-temperature monitoring device to realize the double sensitivity measurement of pressure and temperature, but this structure reduced the pressure measurement accuracy and couldnot achieve the precise temperature compensation by introducing another temperature sensors. Huang et al. [14] designed a diaphragm-type temperature insensitive FBG pressure sensor with a string of two bare FBGs directly bonded on the diaphragm surface. By using two FBGs and analyzing the difference in the wavelength shift, the temperature sensitivity is effectively eliminated. Furthermore, other groups proposed some feasibility techniques such as writing two FBGs in different diameter fibers, using a reference FBG, and combining FBG with Fabry-Perot cavity [15-17]. In this paper, a temperature compensation FBG pressure sensor based on the elastic diaphragm is reported. The mechanical deformation model of the diaphragm is established, and the mathematical relationship between the FBG central wavelength shift and pressure is also reported. Also, the influences of diaphragm's material and dimensions on the sensitivity are numerically analyzed by MATLAB software, and the numerical simulation of the deformation characteristics of the diaphragm is conducted with finite element software ABAQUS. The performance experiments indicated that the FBG pressure sensor has good linearity, low frequency chirp, and high stability, and the temperature cross-sensitivity is effectively avoided, which can provide guidance for the design and manufacture of the sensors, and also can be used to measure pressure in mining engineering, civil engineering, or other complex environment.

\section{Sensor structure and theoretical analysis}

\subsection{Sensor structure}

The schematic diagram of the FBG pressure sensor is shown in Fig. 1. The plane diaphragm and pressure casing are sealed by the o-ring and circlip. One end of the fixed skeleton connects plane diaphragm by welding, and the other end is fixed to the casing. The pressure sensitive FBG (PS FBG) is pasted on the groove in the center of the fixed skeleton by epoxy resin adhesive and used to perceive wavelength shift caused by the pressure. The temperature compensation FBG (TC FBG) is pasted in the casing to compensate temperature, which is connected with the PS FBG through the coupler. Optical fiber sheath and pigtail protection cover are used to encapsulate and protect the optical fiber pigtail.

When the pressure is applied on the plane diaphragm, the deflection deformation of diaphragm will occur and be transferred to the PS FBG through the fixed skeleton, which leads to the shift of the fiber grating central wavelength. The wavelength shifts of TC FBG and PS FBG are detected by a fiber grating analyzer, and we obtain the wavelength 
shift by pressure only when eliminating the wavelength shift caused by temperature and then calculate the pressure value.

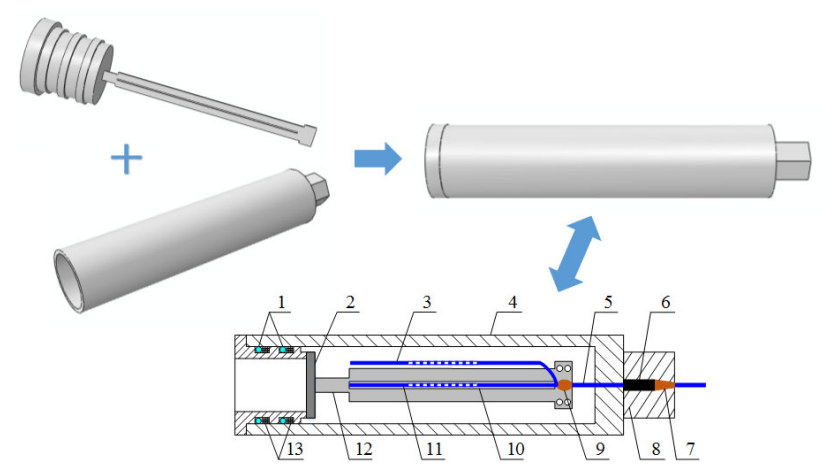

Fig. 1 Schematic diagram of the FBG pressure sensor: 1-o-ring; 2-plane diaphragm; 3-temperature compensation FBG; 4-pressure casing; 5-optical fiber pigtail; 6-optical fiber sheath; 7-pigtail protection cover; 8-nut; 9-coupler; 10-groove; 11-pressure sensitive FBG; 12-fixed skeleton; 13-circlip.

\subsection{FBG sensing principle}

According to the coupled mode theory [18], due to the incident for broadband optical transmission in the optical fiber Bragg grating when generating mode coupling, the Bragg wavelength $\lambda_{B}$ depends both on the physical characteristics of the fiber and geometrical characteristics of the grating as

$$
\lambda_{B}=2 n_{\text {eff }} \Lambda
$$

where $\lambda_{B}$ is the central wavelength of the fiber grating, $n_{\text {eff }}$ is the effective refractive index of the grating in the fiber core, and $\Lambda$ is the grating period [19]. Both $n_{\text {eff }}$ and $\Lambda$ are the main factors of the central wavelength and dependent on the strain and temperature, which lead to the central wavelength shift and affect reliability and sensitivity of the sensor [20]. For common single-mode fiber [21, 22], the Bragg wavelength shift $\Delta \lambda_{B}$ caused by the strain and temperature changes is given as

$$
\Delta \lambda_{B}=\lambda_{B}\left(1-P_{e}\right) \Delta \mathcal{E}+\lambda_{B}(\alpha+\zeta) \Delta T
$$

where $P_{e}=0.22$ is the effective photo-elastic coefficient of the fiber, $\Delta \varepsilon$ is the axial strain change, $\alpha$ is the thermal-expansion coefficient, $\zeta$ is the thermal-optic coefficient, and $\Delta T$ is the temperature variation. According to (2), the FBG can be used for sensing physical parameters such as the strain, temperature, and pressure.

\subsection{Temperature compensation principle}

As is well known, the PS FBG wavelength shifts with the changes in the temperature and pressure, the TC FBG wavelength shift is only affected by the temperature. In the linear range of the grating, the wavelength shifts of the PS FBG and TC FBG with the changes in the temperature and pressure can be described as the following formulae [23, 24]:

$$
\begin{gathered}
\Delta \lambda_{P} / \lambda_{P}=\left(1-P_{e}\right) \Delta \varepsilon+\left(\alpha_{P}+\zeta_{P}\right) \Delta T_{P}= \\
\left(1-P_{e}\right) \Delta \varepsilon+K_{T 1} \Delta T_{P} \\
\Delta \lambda_{T} / \lambda_{T}=\left(\alpha_{T}+\zeta_{T}\right) \Delta T_{T}=K_{T 2} \Delta T_{T}
\end{gathered}
$$

where $\Delta \lambda_{P}$ is the wavelength shift of the PS FBG, $\lambda_{P}$ is the central wavelength of the PS FBG, $\Delta \lambda_{T}$ is the wavelength shift of the TC FBG, $\lambda_{T}$ is the central wavelength of the TC FBG, $K_{T 1}$ is the temperature sensitivity of the PS FBG, and $K_{T 2}$ is the temperature sensitivity of the TC FBG. The PS FBG and TC FBG are located in the same temperature field $\left(\Delta T_{P}\right.$ $\left.=\Delta T_{T}\right)$. Synthesizing (3) and (4), we have

$$
\Delta \lambda_{P} / \lambda_{P}-K_{T 1} \Delta \lambda_{T} / K_{T 2} \lambda_{T}=\left(1-P_{e}\right) \Delta \varepsilon .
$$

According to (5), the effect of temperature variation on the strain measurement can be solved, which overcomes the problem of cross-sensitivity of the strain and temperature.

\subsection{Mechanical analysis of plane diaphragm}

The spring element of the pressure sensor is a plane circular diaphragm, and the strain model is shown in Fig. 2. Deformation and strain would be produced when the pressure liquid to be measured is introduced into the chamber. Based on the small deformation theory $[25,26]$, the differential equation of the plane circular diaphragm can be calculated as

$$
\frac{d}{d r}\left[\frac{1}{r} \frac{d}{d r}\left(r \frac{d \omega}{d r}\right)\right]=\frac{Q}{K}
$$

where $r$ is the distance between the measuring point and the diaphragm center, $\omega$ is the diaphragm deflection, $Q$ is the unit length shear force of the circular cross section with a radius of $r, K=$ 
$E h^{3} / 12\left(1-\mu^{2}\right)$ is the bending stiffness of the diaphragm, $\mu$ is the Poisson's ratio of the diaphragm, $E$ is the elastic modulus of the diaphragm, and $h$ is the diaphragm thickness.

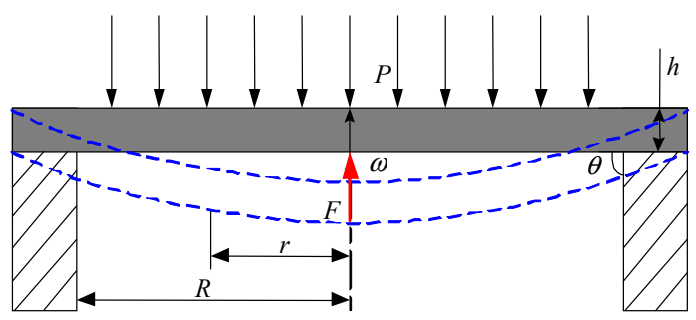

Fig. 2 Strain model for the plane circular diaphragm.

When the uniform pressure $P$ is applied to the surface of the plane circular diaphragm, the shear force can be obtained as follows:

$$
2 \pi r Q=\pi r^{2} P .
$$

Synthesizing (6) and (7), we have

$$
\left\{\begin{array}{l}
\theta=\frac{d \omega}{d r}=\frac{P r^{3}}{16 K}+\frac{r}{2} C_{1}+\frac{1}{r} C_{2} \\
\omega=\frac{P r^{4}}{64 K}+\frac{r^{2}}{4} C_{1}+C_{2} \ln \frac{r}{R}+C_{3}
\end{array}\right.
$$

where $R$ is the diaphragm radius, $\theta$ is the rotation angle, and $\omega$ is the diaphragm deflection, and the boundary conditions are as follows:

$$
\left\{\begin{array}{l}
\left.\theta\right|_{r=0}=0 \\
\left.\theta\right|_{r=R}=0 \\
\left.\omega\right|_{r=R}=0
\end{array}\right.
$$

So we can obtain $C_{1}=-P R^{2} / 8 K, C_{2}=0$, and $C_{3}=$ $P R^{4} / 64 K$. Then the diaphragm deflection $\omega$ can be given as follows:

$$
\omega=\frac{3 P\left(1-\mu^{2}\right)}{16 E h^{3}}\left(R^{4}-2 R^{2} r^{2}+r^{4}\right) .
$$

From (10), when the radius $r=0$, the maximum deflection of the circular plane diaphragm can be expressed as follows:

$$
\omega_{P}=3 P\left(1-\mu^{2}\right) R^{4} / 16 E h^{3} .
$$

Under the pressure, the deflection of the diaphragm induces the concentrated force $F=$ $A E_{f} \Delta L_{f} / L_{f}$ in the fixed skeleton, where $\Delta L_{f}$ is the deformation of the fixed skeleton, $L_{f}$ is the length of the fixed skeleton, $A$ is the cross sectional area of the fixed skeleton, and $E_{f}$ is the Young's modulus of the fixed skeleton. Therefore, the displacement in the center of the diaphragm caused by the force $F$ is expressed as follows [27]:

$$
\omega_{F}=3 F\left(1-\mu^{2}\right) R^{2} / 4 \pi E h^{3} .
$$

The fixed skeleton and PS FBG have the same deformation, that is $\Delta L=\Delta L_{f}$, where $\Delta L$ is the deformation of the PS FBG.

The axial strain $\Delta \varepsilon$ of the PS FBG under diaphragm deflection can be expressed as follows:

$$
\Delta \varepsilon=\Delta L / L=\omega_{P}-\omega_{F} / L .
$$

Synthesizing (5), (11), (12), and (13), the mathematical model between the pressure and wavelength of the sensor can be formulated as

$$
P=\frac{16 E L h^{3}\left(1+\frac{3\left(1-\mu^{2}\right) R^{2} A E_{f}}{4 \pi E h^{3} L_{f}}\right)}{3\left(1-\mu^{2}\right)\left(1-P_{e}\right) R^{4}}\left(\frac{\Delta \lambda_{P}}{\lambda_{P}}-\frac{K_{T 1}}{K_{T 2}} \frac{\Delta \lambda_{T}}{\lambda_{T}}\right)
$$

where $L$ is the effective length of the PS FBG. Thus, the measured pressure value can be obtained from (14).

\section{Numerical analysis and finite element simulation}

\subsection{Effect of material parameters on sensitivity}

Equation (14) shows that the different pressure values of the sensor are affected by any parameters of the diaphragm material, such as the elastic modulus, Poisson's ratio, radius, and thickness. Hence, the sensitivity and measure range can be adjusted by utilizing different materials and sizes of the diaphragm to accommodate different measure environments.

From (14), the sensitivity coefficient $S_{P}$ can be expressed as follows:

$$
S_{P}=\frac{3\left(1-\mu^{2}\right)\left(1-P_{e}\right) R^{4}}{16 E L h^{3}\left(1+\frac{3\left(1-\mu^{2}\right) R^{2} A E_{f}}{4 \pi E h^{3} L_{f}}\right)} .
$$

The effect of material parameters on the sensitivity coefficient are numerically analyzed by MATLAB 
software, as shown in Fig. 3. Figure 3(a) shows that the sensitivity coefficient decreases linearly, the change range is small with an increase in the length

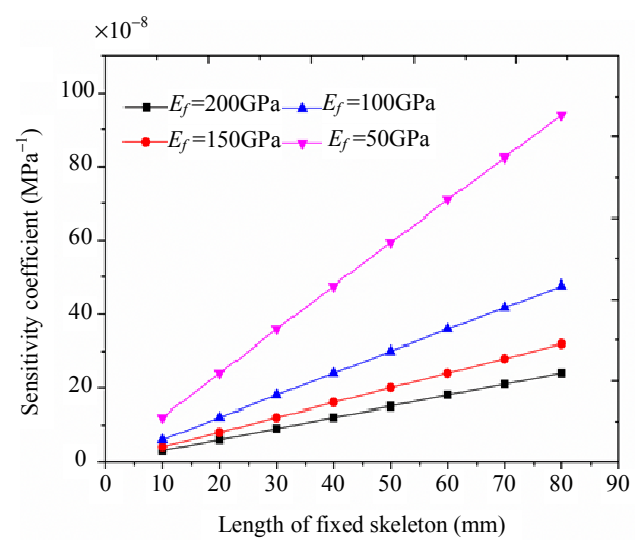

(a)

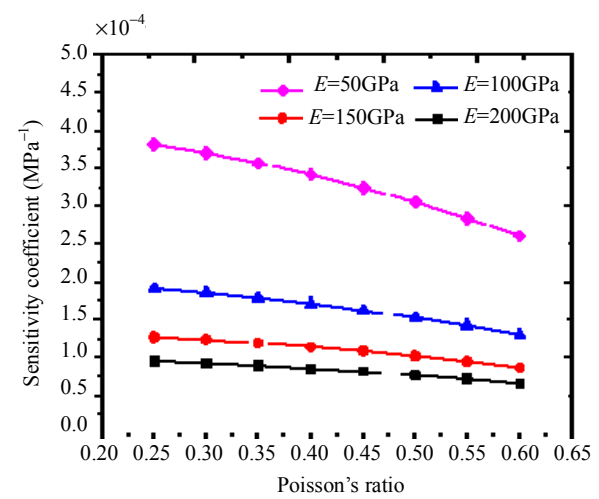

(c)

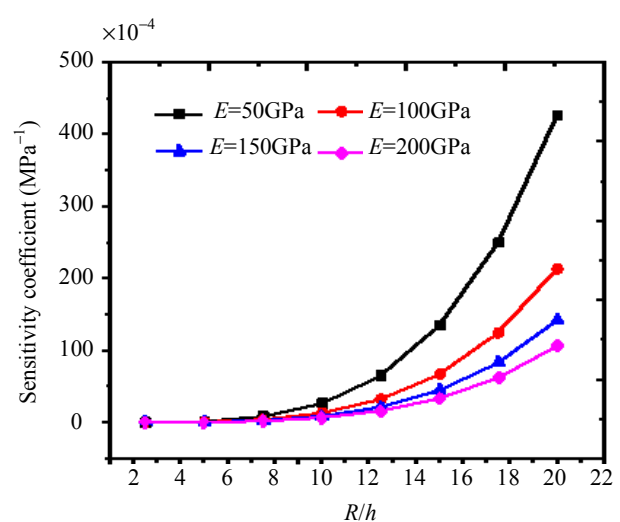

(e) of the fixed skeleton, and the influence of the length of the fixed skeleton on the sensitivity decreases with an increase in the elastic modulus. Figure 3(b)

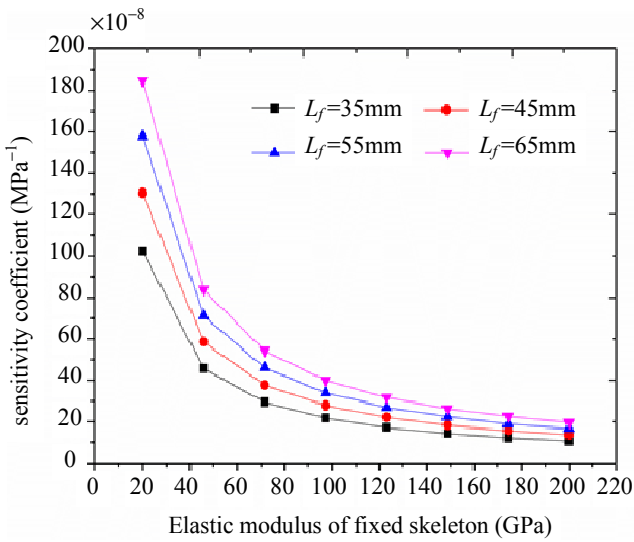

(b)

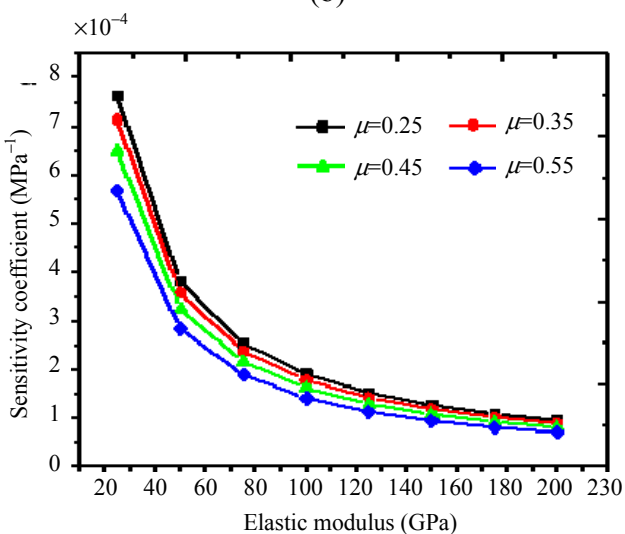

(d)

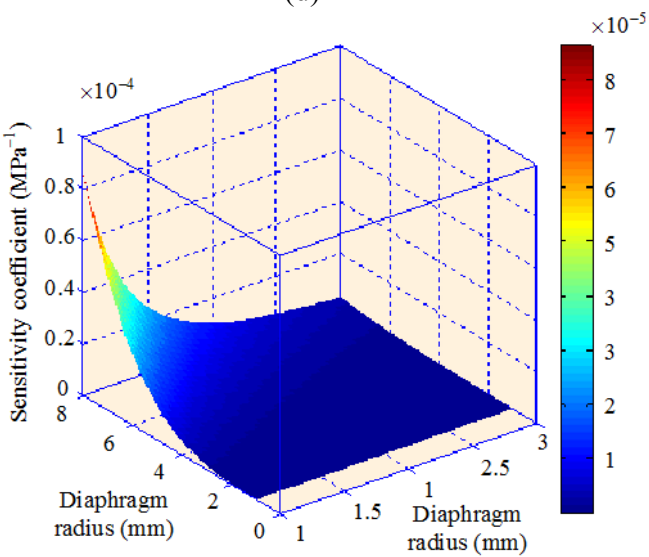

(f)

Fig. 3 Curves of material parameters and sensitivity coefficients: (a) effect of the length of the fixed skeleton on the sensitivity coefficient: calculation parameters: $R=7 \mathrm{~mm}, h=1.2 \mathrm{~mm}, E=195 \mathrm{GPa}, \mu=0.272$, and $L=33 \mathrm{~mm}$, (b) effect of elastic modulus of the fixed skeleton on the sensitivity coefficient: calculation parameters: $R=7 \mathrm{~mm}, h=1.2 \mathrm{~mm}, E=195 \mathrm{GPa}, \mu=0.272$, and $L=33 \mathrm{~mm}$, (c) effect of the Poisson's ratio on the sensitivity coefficient: calculation parameters: $R=7 \mathrm{~mm}, h=1.2 \mathrm{~mm}$, and $L=10 \mathrm{~mm}$, (d) effect of elastic modulus on the sensitivity coefficient: calculation parameters: $R=7 \mathrm{~mm}, h=1.2 \mathrm{~mm}$, and $L=10 \mathrm{~mm}$, (e) effect of diaphragm structure dimension on the sensitivity coefficient: calculation parameters: $h=1 \mathrm{~mm}, L=10 \mathrm{~mm}$, and $\mu=0.3$, and (f) three dimensional curves of sensitivity coefficient with diaphragm radius and thickness: calculation parameters: $E=195 \mathrm{GPa}, \mu=0.272$, and $L=33 \mathrm{~mm}$. 
shows that the sensitivity coefficient inverse proportion function decreases with an increase in the elastic modulus of the fixed skeleton. We can see that the influence of the length and elastic modulus of the fixed skeleton on the sensitivity of the sensor is smaller than those of structure and material parameters of the diaphragm. Figure 3(c) shows that the sensitivity coefficient decreases linearly, the change range is small with an increase in the Poisson's ratio, and the influence of Poisson's ratio on sensitivity decreases with an increase in the elastic modulus. Figure 3(d) shows that the sensitivity coefficient inverse proportion function decreases with an increase in the elastic modulus, and when the elastic modulus is small, the changes range of the sensitivity coefficient is large. Figure 3(e) shows that the sensitivity coefficient increases obviously, and the change range is large with an increase in the structure dimension $R / h$. We can know the diaphragm radius and thickness are the main factors that affect the sensitivity coefficient. We can get a higher sensitivity coefficient by increasing the diaphragm radius and reducing the diaphragm thickness, as shown in Fig. 3(f), which indicates that we should increase the sensitivity and maintain the structure size in the practical optimized design of the

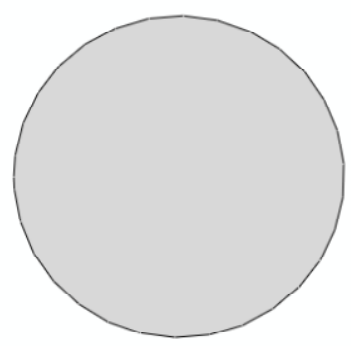

(a)

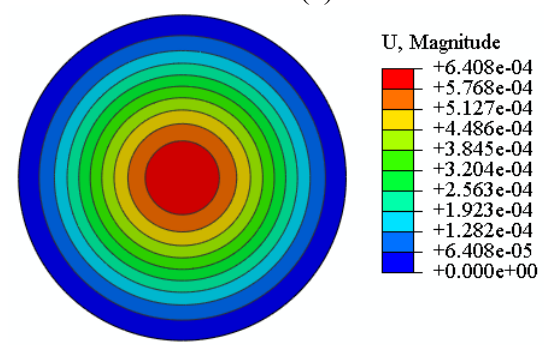

(c) sensor in order to achieve a good performance [28].

\subsection{Finite element simulation of diaphragm}

In order to observe the deformation state and real-time variation of the diaphragm after being subjected to different uniform pressures, the simulation analysis of the diaphragm is conducted with finite element software ABAQUS, and the parameters of the diaphragm used in the simulation are given as follows: diaphragm radius $R=6 \mathrm{~mm}$, diaphragm thickness $h=1 \mathrm{~mm}$, elastic modulus $E=195 \mathrm{GPa}$, and Poisson's ratio $\mu=0.272$. Finally, the solid model, the finite element model, and the displacement clouds of the diaphragm under different uniform pressures of $100 \mathrm{kPa}, 500 \mathrm{kPa}$, $1000 \mathrm{kPa}$, and $2000 \mathrm{kPa}$ are shown in Fig. 4.

It can be found from Fig. 4 that the displacement of the diaphragm increases with an increase in the pressure, and the maximum displacement locates in the diaphragm center and decreases along the radius direction. The displacement of the diaphragm center reaches $1.282 \times 10^{-3} \mathrm{~mm}$ under $1000 \mathrm{kPa}$, resulting in the same change in the FBG length. The effective length of the PS FBG $L$ is $33 \mathrm{~mm}$, and the strain sensitivity of the bare FBG is $1.2 \mathrm{pm} / \mu \varepsilon$ ( $\mu \varepsilon$ is the micro-strain). Accordingly, the central wavelength shift of the PS FBG is $46.62 \mathrm{pm}$, that is, the pressure sensitivity is $46.62 \mathrm{pm} / \mathrm{MPa}$.

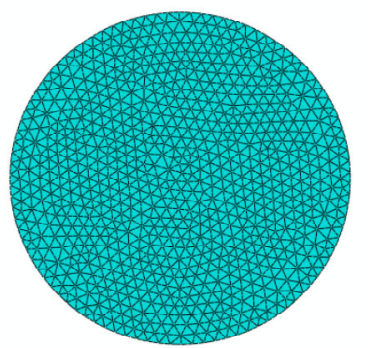

(b)

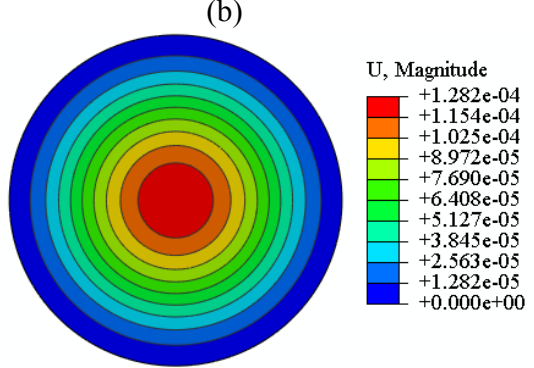

(d) 


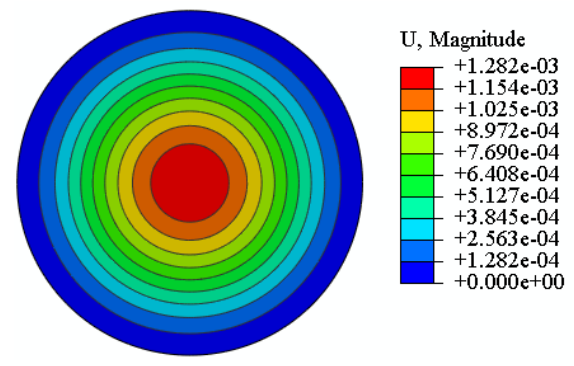

(e)

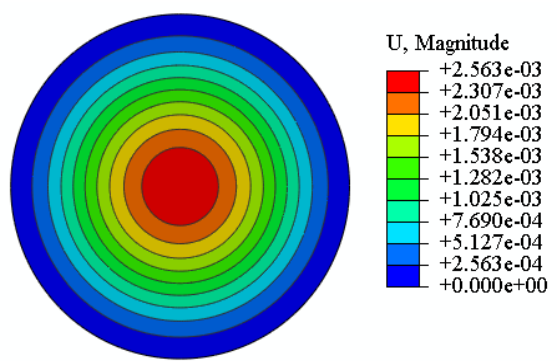

(f)

Fig. 4 Model and displacement clouds of the diaphragm: (a) solid model of the diaphragm, (b) finite element model of the diaphragm, (c) displacement cloud of the diaphragm under $100 \mathrm{kPa}$, (d) displacement cloud of the diaphragm under $500 \mathrm{kPa}$, (e) displacement cloud of the diaphragm under $1000 \mathrm{kPa}$, and (f) displacement cloud of the diaphragm under $2000 \mathrm{kPa}$.

\section{Sensor experiment and result analysis}

\subsection{Sensor fabrication}

The reasonable choice of the elastic diaphragm material is the basis for the design and fabrication of the sensor [29]. FBG pressure sensor design principles are as follows:

(1) The material has a high strength, high elastic limit, and small temperature coefficient within the measurement scope;

(2) The material has a good anti-oxidation, anti-causticity, and impact toughness;

(3) The material is isotropic which has a good heat treatment performance and small thermal-expansion coefficient;

(4) Considering the measurement range and structure size, select the suitable adhesive. The FBG pressure sensor's shell and diaphragm adopt stainless steel 17-4PH and use epoxy resin adhesive gratin. The photo of the sensor is shown in Fig.5.

The fiber grating parameters used in the sensor are as follows: the effective length of the FBG $L=33 \mathrm{~mm}$, the central wavelength of the PS FBG $\lambda_{P}=$ $1528.275 \mathrm{~nm}$, and the central wavelength of the TC FBG $\lambda_{T}=1531.383 \mathrm{~nm}$. The pre-tensioning effect of the packaging process causes a wavelength shift of $1 \mathrm{~nm}$. And the wavelength of the PS FBG after stabilization is $1529.298 \mathrm{~nm}$. When the temperature changes is small, that is $\Delta \lambda_{T}=0$, the theoretical pressure sensitivity can be calculated, which is $40.43 \mathrm{pm} / \mathrm{MPa}$.

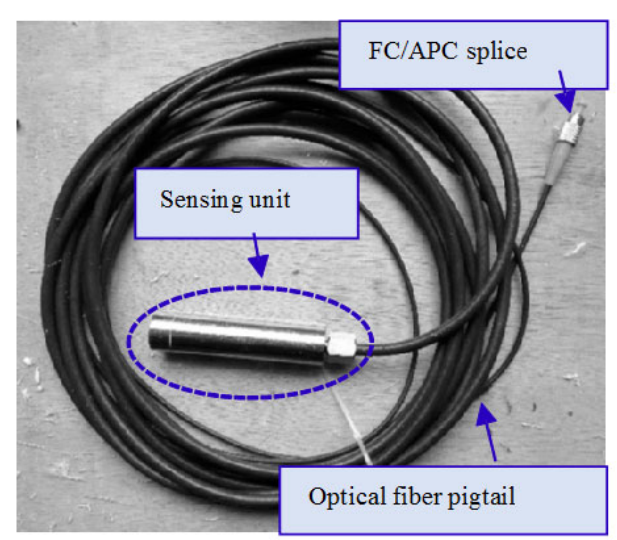

Fig. 5 Photo of the FBG pressure sensor.

\subsection{Sensor measurement system}

The pressure experiment measurement system is shown in Fig. 6. The pressure loading device (SSR-YBS-60TB) with the maximum range is $60 \mathrm{MPa}$, and the accuracy is $0.03 \mathrm{MPa}$. The FBG pressure sensor is connected to the pressure loading device. The optical fiber pigtail of the sensor is connected to the FBG analyzer by the FC/APC (ferrule connector/angled physical contact) splice, and the FBG analyzer is connected to the computer processing system by the network cable. The pressure source loading is controlled by the handle, the size of pressure of the piston cylinder is controlled by pressure retaining valves and handle, the digital pressure gauge displays pressure value in real time, and the FBG analyzer modulates and demodulates the pressure and displays the dynamic spectrum in the computer processing system in real time. 
The temperature experiment measurement system is mainly composed of the broadband light source, FBG analyzer, temperature measuring device, computer processing system, and optical fiber, which has the same wavelength demodulation and data processing with the pressure experiment system. The temperature measuring device (RTS-40) with the measuring change is from $-40{ }^{\circ} \mathrm{C}$ to $95^{\circ} \mathrm{C}$, the temperature resolution is $0.01{ }^{\circ} \mathrm{C}$, and the precision is $0.05^{\circ} \mathrm{C}$. The FBG analyzer wavelength scanning range is $1510 \mathrm{~nm}-1590 \mathrm{~nm}$, the wavelength resolution is $1 \mathrm{pm}$, the working frequency is $1 \mathrm{~Hz}-10 \mathrm{~Hz}$, and the sampling frequency is $1 \mathrm{~Hz}$.

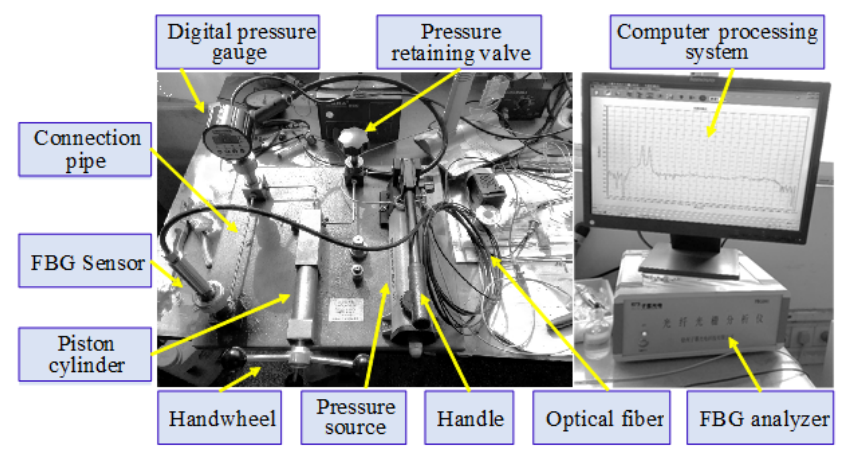

Fig. 6 Photo of the pressure experiment measurement system.

\subsection{Experiment and result}

\subsubsection{Pressure experiment}

In the pressure experiment process, the pressure sensor is loaded and controlled by the piston cylinder and hand-wheel, with pressure changed from $0 \mathrm{MPa}$ to $50 \mathrm{MPa}$ at an interval of $5 \mathrm{MPa}$, and the temperature is fixed at the room temperature $\left(25^{\circ} \mathrm{C}\right)$, then we record the central wavelength shift of the PS FBG and TC FBG for each step. Figure 7 shows the central wavelength changes versus the pressure variation for the PS FBG and TC FBG. The wavelength of the PS FBG has a blue shift, indicating that the PS FBG is compressed and senses the negative strain. While the wavelength of the TC FBG has a red shift which verifies the TC FBG is not sensitive to the pressure.

It can be found from Fig. 7 that the central wavelength of the PS FBG decreases linearly with an increase in the pressure and has a negative linear correlation. The linear fitting equation $y=-0.0357 x+$ 1529.298 is also presented, the linear fitting correlation coefficient reaches $99.95 \%$, and the pressure sensitivity is $35.7 \mathrm{pm} / \mathrm{MPa}$. This pressure sensitivity is slightly smaller than the theoretical value and simulation value, which is mainly caused by:

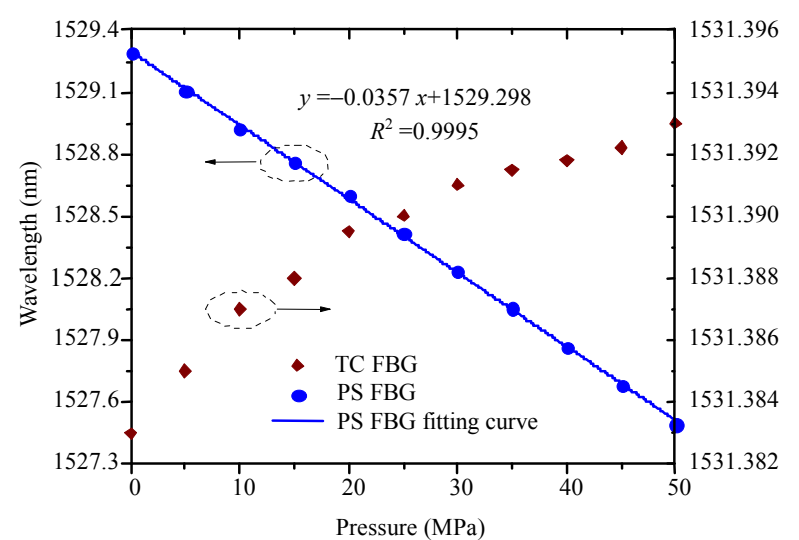

Fig. 7 Central wavelength changes of the two FBGs versus pressure variation.

(1) The errors of the structure size and bad processing quality of sensor's parts in the sensor fabrication, which affect the pressure sensitivity;

(2) The effect of the PS FBG and the elastic diaphragm center, which are not in the same axis;

(3) The elastic plastic character of the epoxy resin, which leads to the strain transfer efficiency between the diaphragm and the FBG does not reach $100 \%$;

(4) Some boundary conditions in the simulation process, which are idealized and have difference from the actual ones;

(5) The fixed skeleton in the casing, which gives the diaphragm a reaction force, which reduces the diaphragm displacement and affects the pressure sensitivity.

\subsubsection{Temperature experiment}

During the temperature experiment, the 
temperature is controlled by the temperature measuring device and applied to the sensor, which is changed from $-10{ }^{\circ} \mathrm{C}$ to $70{ }^{\circ} \mathrm{C}$ with a step of $10{ }^{\circ} \mathrm{C}$ and without applying any pressure, and then we record the central wavelength of the PS FBG and TC FBG for each step. Figure 8 shows the central wavelength changes versus temperature variation for the PS FBG and TC FBG. The central wavelengths of the PS FBG and TC FBG increase linearly with an increase in the temperature and have a positive linear correlation. The linear fitting results are presented that the linear fitting correlation coefficient reaches $99.95 \%$ and $99.94 \%$, respectively. The temperature sensitivities of the PS FBG and TC FBG are $K_{T 1}=1.63 \mathrm{pm} /{ }^{\circ} \mathrm{C}$ and $K_{T 2}=$ $11.25 \mathrm{pm} /{ }^{\circ} \mathrm{C}$, respectively.

In order to evaluate the sensor's temperature compensation performance, the variation of wavelength difference $\left(\Delta \lambda_{P} / \lambda_{P}-\Delta \lambda_{T} \cdot K_{T 1} / \lambda_{T} \cdot K_{T 2}\right)$ versus pressure increase has been given, as shown in Fig. 9. The linear fitting results are obtained. The linear fitting correlation coefficient reaches $99.83 \%$, and the sensitivity coefficient is $2.35 \times 10^{-5} / \mathrm{MPa}$. Therefore, the actual pressure sensitivity of the sensor is calculated to be $37.48 \mathrm{pm} / \mathrm{MPa}$, and the sensor's temperature compensation effect is conspicuous.

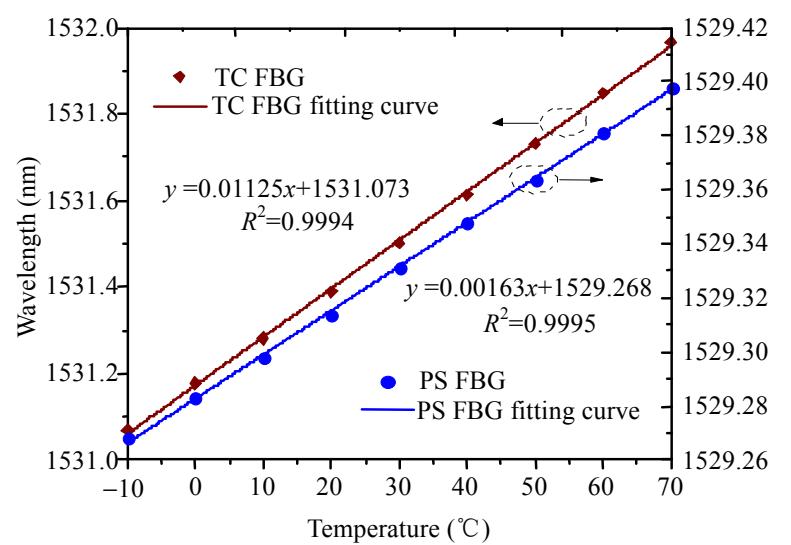

Fig. 8 Central wavelength changes of the two FBGs versus temperature variation.

\subsubsection{Stability experiment}

The pressure is applied to the FBG pressure sensor of $0 \mathrm{MPa}, 22 \mathrm{MPa}$, and $44 \mathrm{MPa}$, the temperature is fixed at the room temperature, and the bandwidths of $3 \mathrm{~dB}$ reflection spectrum of the PS FBG are $0.438 \mathrm{~nm}, 0.439 \mathrm{~nm}$, and $0.436 \mathrm{~nm}$, respectively, indicating that there is no bandwidth broadening or low frequency chirp phenomenon in the reflection spectrum of the sensor, as shown in Fig. 10.

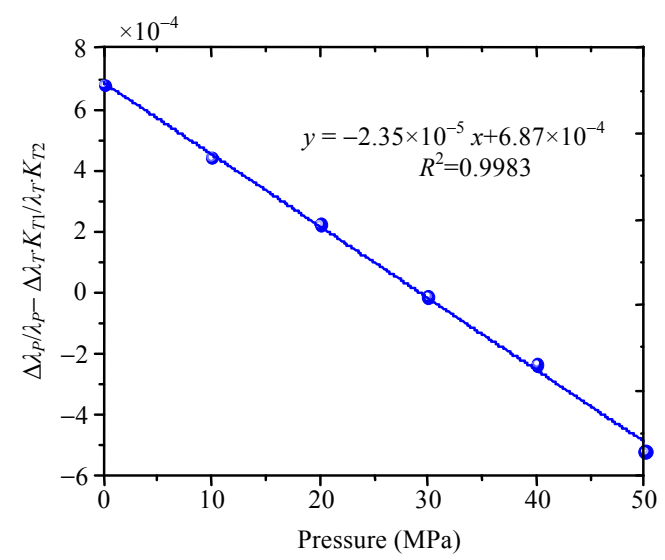

Fig. 9 Variation of wavelength difference $\left(\Delta \lambda_{P} / \lambda_{P}-\right.$ $\Delta \lambda_{T} \cdot K_{T 1} / \lambda_{T} \cdot K_{T 2}$ ) versus pressure increase.

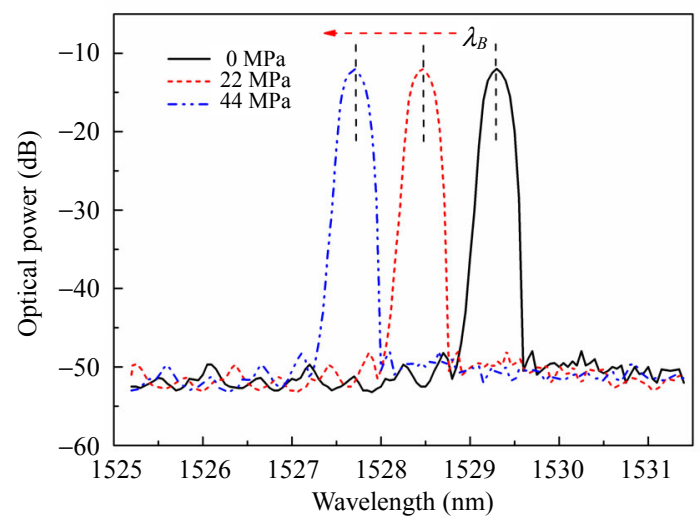

Fig. 10 Reflection spectra of the sensor under different pressures of $0 \mathrm{MPa}, 22 \mathrm{MPa}$, and $44 \mathrm{MPa}$.

Stability experiment has been carried out with the pressure, which is applied to the pressure sensor of $20 \mathrm{MPa}$, and the temperature is fixed at the room temperature $\left(25^{\circ} \mathrm{C}\right)$ for 10 times. We record the stable central wavelength of the PS FBG, and the results are listed in Table 1 . The mean square deviation of wavelength can be calculated is $3.2 \times$ $10^{-3}$, which shows that the variation of the sensor's central wavelength is small and has high stability performance with the same pressure. 
Table 1 Experimental results for wavelength of the PS FBG.

\begin{tabular}{ccccc}
\hline & Times & Wavelength $(\mathrm{nm})$ & Times & Wavelength $(\mathrm{nm})$ \\
\hline & 1 & 1528.597 & 6 & 1528.602 \\
$\left(25^{\circ} \mathrm{C}\right.$, & 2 & 1528.595 & 7 & 1528.593 \\
$20 \mathrm{MPa})$ & 3 & 1528.601 & 8 & 1528.599 \\
& 4 & 1528.598 & 9 & 1528.595 \\
& 5 & 1528.594 & 10 & 1528.592 \\
\hline
\end{tabular}

From the above research, it could be concluded that the FBG pressure sensor with temperature compensation based on the plane diaphragm can be used to measure pressure of gas and liquid. By analyzing the experimental data, the sensor has the good linearity, low frequency chirp, and high stability. The temperature cross-sensitivity is effectively avoided, which can provide guidance for the design and manufacture of the sensors. With these advantages, this kind of FBG pressure sensor is supposed to have many engineering applications to the pressure measurement in mining engineering, civil engineering or other complex environment.

\section{Conclusions}

In this paper, a temperature compensation FBG pressure sensor based on the plane diaphragm has been designed and tested. The sensor adopts a plane circular diaphragm as the pressure sensitive component, and the mechanical deformation model of the diaphragm is established. The mathematical relationship between the FBG central wavelength shift and pressure is deduced by the theoretical calculation. The effects of diaphragm material parameters on the sensitivity coefficient are numerically analyzed by MATLAB software, and the theoretical pressure sensitivity of $40.43 \mathrm{pm} / \mathrm{MPa}$ is achieved. Furthermore, the sensitivity and measure range can be adjusted by utilizing the different materials and sizes of the diaphragm to accommodate different measurement environments. In addition, the deformation characteristics of the diaphragm under different uniform pressures are conducted with numerical simulation by finite element software ABAQUS, and the results show that the pressure sensitivity is $46.62 \mathrm{pm} / \mathrm{MPa}$. The performance experiments indicates that the pressure sensitivity of the sensor is $35.7 \mathrm{pm} / \mathrm{MPa}$ which is slightly smaller than the theoretical value and simulation value, and has good linearity with a linear fitting correlation coefficient of $99.95 \%$. More importantly, the actual pressure sensitivity of the sensor is $37.48 \mathrm{pm} / \mathrm{MPa}$ after temperature compensation, and the sensor has the advantages of low frequency chirp and high stability, which can be used to measure pressure in mining engineering, civil engineering, or other complex environment.

\section{Acknowledgment}

This study was financially supported by the Fundamental Research Funds for the Central Universities (No. 2017CXNL01), the Funded Project of Jiangsu Provincial Six Talent Peaks (No. 2014-ZBZZ-008), and a Project Funded by the Priority Academic Program Development of Jiangsu Higher Education Institutions (No. SZBF2011-6-B35).

Open Access This article is distributed under the terms of the Creative Commons Attribution 4.0 International License (http://creativecommons.org/ licenses/by/4.0/), which permits unrestricted use, distribution, and reproduction in any medium, provided you give appropriate credit to the original author(s) and the source, provide a link to the Creative Commons license, and indicate if changes were made.

\section{References}

[1] S. J. Mihailov, "Fiber Bragg grating sensors for harsh environments," Sensors, 2012, 12(2): 1898-1918.

[2] B. Lee, "Review of the present status of optical fiber sensors," Optical Fiber Technology, 2003, 9(2): $57-59$.

[3] H. Tsuda, "Fiber Bragg grating vibration-sensing system, insensitive to Bragg wavelength and employing fiber ring laser," Optics Letters, 2010, 35(14): 2349-2351.

[4] A. P. Zhang, B. O. Guan, X. M. Tao, and H. Y. Tam, "Experimental and theoretical analysis of fiber Bragg gratings under lateral compression," Optics Communications, 2002, 206(1-3): 81-87.

[5] D. Song, J. Zou, Z. Wei, and H. L. Cui. "High-sensitivity fiber Bragg grating pressure sensor 
using metal bellows," Optics Engineering, 2009, 48(3): 034403-1-034403-3.

[6] B. J. Peng, Y. Zhao, J. Yang, and M. Zhao, "Pressure sensor based on a free elastic cylinder and birefringence effect on an FBG with temperature-compensation," Measurement, 2005, 38(2): 176-180.

[7] Y. Zhang, Z. G. Liu, Z. Y. Guo, S. Z. Yuan, D. J. Feng, X. Y. Dong, et al., "A high-sensitivity fiber grating pressure sensor and its pressure sensing characteristics," Acta Optica Sinica, 2002, 22(1): 89-91.

[8] Y. Zhao, C. B. Yu, and Y. B. Liao, "Differential FBG sensor for temperature compensated high-pressure (or displacement) measurement," Optics \& Laser Technology, 2004, 36(1): 39-42.

[9] Y. Zhao, Y. Zhao, and M. G. Zhao, "Novel force sensor based on a couple of fiber Bragg gratings," Measurement, 2005, 38(1): 30-33.

[10] D. Sengupta, M. S. Shankar, P. S. Reddy, R. L. N. S. Prasad, and K. Srimannarayana, "Sensing of hydrostatic pressure using FBG sensor for liquid level measurement," Microwave \& Optical Technology Letters, 2012, 7(54): 1679-1683.

[11] H. Ahmad, S. W. Harun, W. Y. Chong, M. Z. Zulkifli, M. M. M. Thant, Z. Yusof, et al., "High-sensitivity pressure sensor using a polymer embedded FBG," Microwave \& Optical Technology Letters, 2008, 50(1): 60-61.

[12] L. H. Liu, H. Zhang, Q. D. Zhao, Y. L. Liu, and F. Li, "Temperature-independent FBG pressure sensor with high sensitivity," Optical Fiber Technology, 2007, 13(1): 78-80.

[13] H. L. Pan, H. J. Dong, G. Y. Zhang, and J. He, "Research on fiber grating pressure/temperature monitoring device of distinguish range and double sensitivity," Chinese Journal of Lasers, 2013, 40(2): 0205005-1-0205005-6.

[14] J. Huang, Z. D. Zhou, X. Y. Wen, and D. S. Zhang, "A diaphragm-type fiber Bragg grating pressure sensor with temperature compensation," Measurement Journal of the International Measurement Confederation, 2013, 46(3): 1041-1046.

[15] H. J. Sheng, W. F. Liu, K. R. Lin, S. S. Bor, and M. Y. Fu, "High-sensitivity temperature-independent differential pressure sensor using fiber Bragg gratings," Optics Express, 2008, 16(20): 16013-1-16013-8.

[16] Y. S. Hsu, L. Wang, W. F. Liu, and Y. J. Chiang, "Temperature compensation of optical fiber Bragg grating pressure sensor," IEEE Photonics Technology Letters, 2006, 18(7): 874-876.

[17] W. H. Wang, X. S. Jiang, and Q. X. Yu, "Temperature self-compensation fiber-optic pressure sensor based on fiber Bragg grating and Fabry-Perot interference multiplexing," Optics Communications, 2012, 285(16): 3466-3470.

[18] A. Cusano, A. Cutolo, J. Nasser, M. Giordano, and A. Calabrò, "Dynamic strain measurements by fibre Bragg grating sensor," Sensors \& Actuators A: Physical, 2004, 110(1): 276-281.

[19] B. Torres, I. Payá-Zaforteza, P. A. Calderón, and J. M. Adam, "Analysis of the strain transfer in a new FBG sensor for structural health monitoring," Engineering Structures, 2011, 33(2): 539-548.

[20] X. F. Yang, S. J. Luo, Z. H. Chen, J. H. Ng, and C. Lu, "Fiber Bragg grating strain sensor based on fiber laser," Optics Communications, 2007, 271(1): 203-206.

[21] S. C. Tao, X. P. Dong, and B. W. Lai, "Temperature-insensitive fiber Bragg grating displacement sensor based on a thin-wall ring," Optics Communications, 2016, 372: 44-48.

[22] J. P. He, Z. Zhou, and J. P. Ou, "Simultaneous measurement of strain and temperature using a hybrid local and distributed optical fiber sensing system," Measurement, 2014, 47(1): 698-706.

[23] S. M. Wang, Y. J. Chiang, and L. Wang, "New structure for multipoint temperature-independent strain sensing system employing optical power detection scheme," Optical Fiber Technology, 2008, 14(2): 167-171.

[24] J. Yang, Y. Zhao, B. J. Peng, and X. Wan, "Temperature-compensated high pressure FBG sensor with a bulk-modulus and self-demodulation method," Sensors \& Actuators A: Physical, 2005, 118(2): 254-258.

[25] W. T. Zhang, F. Li, and Y. L. Liu, "FBG pressure sensor based on the double shell cylinder with temperature compensation," Measurement, 2009, 42(3): 408-411.

[26] G. Allwood, G. Wild, A. Lubansky, and S. Hinckley, "A highly sensitive fiber Bragg grating diaphragm pressure transducer," Optical Fiber Technology, 2015, 25: 25-32.

[27] V. R. Pachava, S. Kamineni, S. S. Madhuvarasu, and K. Putha, "A high sensitive FBG pressure sensor using thin metal diaphragm," Journal of Optics, 2014, 43(2): 117-121.

[28] H. Bao, X. Dong, C. Zhao, L. Y. Shao, C. C. Chan, and P. Shum, "Temperature-insensitive FBG tilt sensor with a large measurement range," Optics Communications, 2009, 283(6): 968-970.

[29] S. Jiang, J. Wang, and Q. Sui, "Distinguishable circumferential inclined direction tilt sensor based on fiber Bragg grating with wide measuring range and high accuracy," Optics Communications, 2015, 355: 58-63. 